\title{
Transmission Character of General Function Photonic Crystals
}

\author{
Xiang-Yao $\mathrm{Wu}^{a}$, Bo-Jun Zhang ${ }^{a}$, Jing-Hai Yang ${ }^{a}$, Si-Qi Zhang ${ }^{a}$ \\ Xiao-Jing Liu ${ }^{a}$, Jing $\mathrm{Wang}^{a}$, Nuo $\mathrm{Ba}^{a}$ and Zhong Hua ${ }^{a}$ \\ ${ }^{a}$ Institute of Physics, Jilin Normal University, Siping 136000, China
}

\begin{abstract}
In the paper, we present a new general function photonic crystals (GFPCs), which refractive index of medium is a arbitrary function of space position. Unlike conventional photonic crystals (PCs), which structure grow from two mediums $A$ and $B$, with different constant refractive indexes $n_{a}$ and $n_{b}$. Based on Fermat principle, we give the motion equations of light in one-dimensional GFPCs, and calculate its transfer matrix, which is different from the conventional PCs. We choose the linearity refractive index function for two mediums $A$ and $B$, and find the transmissivity of one-dimensional GFPCs can be much larger or smaller than 1 for different slope linearity refractive index function, which is different from the transmissivity of conventional PCs (its transmissivity is in the range of 0 and 1). Otherwise, we study the effect of different incident angles, the number of periods and optical thickness on the transmissivity, and obtain some new results different from the conventional PCs.
\end{abstract}

PACS: 42.70.Qs, 78.20.Ci, 41.20.Jb

Keywords: General Photonic crystals; Dispersion relation; Transmissivity

PACS numbers:

\section{Introduction}

Photonic crystals (PCs) are composite structures with a periodic arrangement of materials with different refractive indices in one-dimension (1D), two-dimension (2D) or three-dimension (3D).Due to the introduced periodicity, multiple Bragg scatterings from each unit cell may open a photonic band gaps (PBGs), analogous to the electronic band gaps in semiconductors, with in which the propagation of electromagnetic (EM) waves is completely forbidden. The existence of PBGs will lead to many interesting phenomena, e.g., modification of spontaneous emission [1-5] and photon localization [6-10]. Thus numerous applications of photonic crystals have been proposed in improving the performance of optoelectronic and microwave devices such as high-efficiency semiconductor lasers, right emitting diodes, wave guides, optical filters, high-Q resonators, antennas, frequency-selective surface, optical limiters and amplifiers [11-14]. These applications would be significantly enhanced if the band structure of the photonic crystal could be tuned.

For the conventional PCs, the photonic band gaps remain fixed once the PCs have been fabricated. If the band gaps of the photonic crystals could be tuned the applications would be significantly enhanced. A practical scheme for tuning the band gap was proposed by Busch and John [15]. It has been demonstrated theoretically and experimentally that PCs with liquid crystal infiltration exhibit tunability on applying an external electric field [15]or changing the temperature [16-20]. If the constituent materials of PCs have magnetic permeabilities dependent on the external magnetic field, the photonic band gaps [PBGs] can be altered by changing the external magnetic field [21-28]. An electric or magnetic field changes the PBGs easily than the temperature.

In Ref. [29], we have proposed special function photonic crystals, which the medium refractive index is the function of space position, but the function value of refractive index is equal at two endpoints of every medium $A$ and $B$, and obtain some new results different from the the conventional PCs. In this paper, we present a new general function photonic crystals (GFPCs), which refractive index is a arbitrary function of space position (needless refractive index same at two endpoint). Unlike conventional photonic crystals (PCs), which structure grow from two materials, $\mathrm{A}$ and $\mathrm{B}$, with different dielectric constants $\varepsilon_{A}$ and $\varepsilon_{B}$. Firstly, we give the motion equation of light in one-dimensional GFPCs according to Fermat principle. Secondly, we calculate the transfer matrix for the one-dimensional GFPCs, which is different from the transfer matrix of the conventional PCs. Finally, we give the dispersion relation, band gap structure and transmissivity. We choose the linearity refractive index function for two medium $A$ and $B$, and find the transmissivity of GFPCs can be much larger or smaller than 1 for the different slope linearity refractive index function, which is different from the transmissivity of conventional PCs (its transmissivity is in the range of 0 and 1 ). Otherwise, we study the effect of different incident angles, the number of periods and optical thickness on the transmissivity, and obtain some new results. By the calculation, we find the conventional PCs is the special case of the GFPCs.

\section{The light motion equation in general func- tion photonic crystals}

For the general function photonic crystals, the medium refractive index is a periodic function of the space position, which can be written as $n(z), n(x, z)$ and $n(x, y, z)$ corresponding to one-dimensional, two-dimensional and three-dimensional function photonic crystals. In the following, we shall deduce the light motion equations of the one-dimensional general function photonic crystals, i.e., 


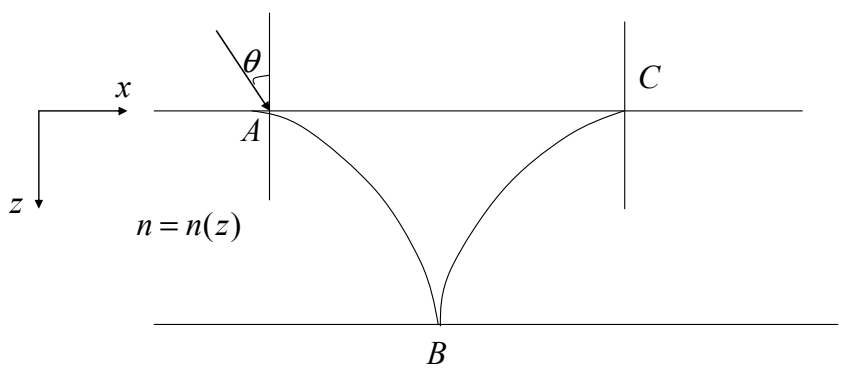

FIG. 1: The motion path of light in the medium of refractive index $n(z)$.

the refractive index function is $n=n(z)$, meanwhile motion path is on $x z$ plane. The incident light wave strikes plane interface point $A$, the curves $A B$ and $B C$ are the path of incident and reflected light respectively, and they are shown in FIG. 1.

The light motion equation can be obtained by Fermat principle, it is

$$
\delta \int_{A}^{B} n(z) d s=0
$$

In the two-dimensional transmission space, the line element $d s$ is

$$
d s=\sqrt{(d x)^{2}+(d z)^{2}}=\sqrt{1+\dot{z}^{2}} d x,
$$

where $\dot{z}=\frac{d z}{d x}$, then Eq. (1) becomes

$$
\delta \int_{A}^{B} n(z) \sqrt{1+(\dot{z})^{2}} d x=0 .
$$

The Eq. (3) change into

$$
\int_{A}^{B}\left(\frac{\partial\left(n(z) \sqrt{1+\dot{z}^{2}}\right)}{\partial z} \delta z+\frac{\partial\left(n(z) \sqrt{1+\dot{z}^{2}}\right)}{\partial \dot{z}} \delta \dot{z}\right) d x=0
$$

At the two end points $A$ and $B$, their variation is zero, i.e., $\delta z(A)=\delta z(B)=0$. For arbitrary variation $\delta z$, the Eq. (4) becomes

$$
\begin{aligned}
& \frac{d n(z)}{d z} \sqrt{1+\dot{z}^{2}}-\frac{d n(z)}{d z} \dot{z}^{2}\left(1+\dot{z}^{2}\right)^{-\frac{1}{2}} \\
& -n(z) \frac{\ddot{z} \sqrt{1+\dot{z}^{2}}-\dot{z}^{2} \ddot{z}\left(1+\dot{z}^{2}\right)^{-\frac{1}{2}}}{1+\dot{z}^{2}}=0,
\end{aligned}
$$

simplify Eq. (5), we have

$$
\frac{d n(z)}{n(z)}=\frac{\dot{z} d \dot{z}}{1+\dot{z}^{2}}
$$

The Eq. (6) is light motion equation in one-dimensional function photonic crystals.

3. The transfer matrix of one-dimensional general function photonic crystals
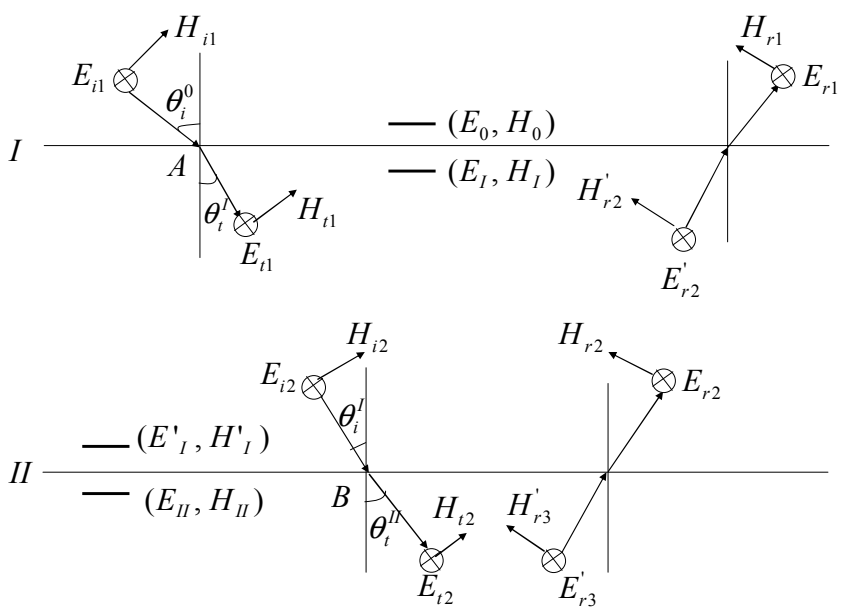

FIG. 2: The light transmission and electric magnetic field distribution figure in FIG.1 medium.

In this section, we should calculate the transfer matrix of one-dimensional general function photonic crystals. In fact, there is the reflection and refraction of light at a plane surface of two media with different dielectric properties. The dynamic properties of the electric field and magnetic field are contained in the boundary conditions: normal components of $D$ and $B$ are continuous; tangential components of $E$ and $H$ are continuous. We consider the electric field perpendicular to the plane of incidence, and the coordinate system and symbols as shown in FIG. 2 .

On the two sides of interface I, the tangential components of electric field $E$ and magnetic field $H$ are continuous, there are

$$
\left\{\begin{array}{l}
E_{0}=E_{I}=E_{t 1}+E_{r 2}^{\prime} \\
H_{0}=H_{I}=H_{t 1} \cos \theta_{t}^{I}-H_{r 2}^{\prime} \cos \theta_{t}^{I} .
\end{array}\right.
$$

On the two sides of interface II, the tangential components of electric field $E$ and magnetic field $H$ are continuous, and give

$$
\left\{\begin{array}{l}
E_{I I}=E_{I}^{\prime}=E_{i 2}+E_{r 2} \\
H_{I I}=H_{I}^{\prime}=H_{i 2} \cos \theta_{i}^{I}-H_{r 2} \cos \theta_{i}^{I},
\end{array}\right.
$$

the electric field $E_{t 1}$ is

$$
E_{t 1}=\left.E_{t 10} e^{i\left(k_{x} x_{A}+k_{z} z\right)}\right|_{z=0}=E_{t 10} e^{i \frac{\omega}{c} n(0) \sin \theta_{t}^{I} x_{A}},
$$

and the electric field $E_{i 2}$ is

$$
\begin{aligned}
E_{i 2} & =\left.E_{t 10} e^{i\left(k_{x}^{\prime} x_{B}+k_{z}^{\prime} z\right)}\right|_{z=b} \\
& =E_{t 10} e^{i \frac{\omega}{c} n(b)\left(\sin \theta_{i}^{I} x_{B}+\cos \theta_{i}^{I} b\right)} .
\end{aligned}
$$

Where $x_{A}$ and $x_{B}$ are $x$ component coordinates corresponding to point $A$ and point $B$. We should give the relation between $E_{i 2}$ and $E_{t 1}$. By integrating the two 


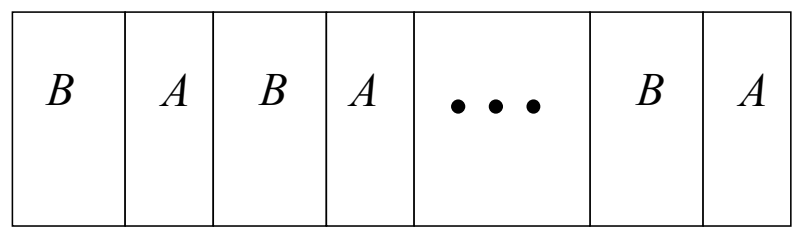

FIG. 3: The structure $(B A)^{N}$ of the general function photoni crystals.

sides of Eq. (6), we can obtain the coordinate compo nent $x_{B}$ of point $B$

$$
\int_{n(0)}^{n(z)} \frac{d n(z)}{n(z)}=\int_{k_{0}}^{k_{z}} \frac{\dot{z} d \dot{z}}{1+\dot{z}^{2}}
$$

to get

$$
k_{z}^{2}=\left(1+k_{0}^{2}\right)\left(\frac{n(z)}{n(0)}\right)^{2}-1
$$

and

$$
d x=\frac{d z}{\sqrt{\left(1+k_{0}^{2}\right)\left(\frac{n(z)}{n(0)}\right)^{2}-1}} .
$$

where $k_{0}=\cot \theta_{t}^{I}$ and $k_{z}=\frac{d z}{d x}$ From Eq. (12), there is $n(z)>n(0) \sin \theta_{t}^{I}$. and the coordinate $x_{B}$ is

$$
x_{B}=x_{A}+\int_{0}^{b} \frac{d z}{\sqrt{\left(1+k_{0}^{2}\right)\left(\frac{n(z)}{n(0)}\right)^{2}-1}}
$$

where $b$ is the medium thickness of FIG. 1 and FIG. 2. By substituting Eqs. (9) and (14)into (10), and using the equality

$$
n(0) \sin \theta_{t}^{I}=n(b) \sin \theta_{i}^{I}
$$

we have

$$
E_{i 2}=E_{t 1} e^{i \delta_{b}}
$$

where

$$
\delta_{b}=\frac{\omega}{c} n_{b}(b)\left(\cos \theta_{i}^{I} b+\sin \theta_{i}^{I} \int_{0}^{b} \frac{d z}{\sqrt{\frac{n_{b}^{2}(z)}{n_{0}^{2} \sin ^{2} \theta_{i}^{0}}-1}}\right),
$$

and similarly

$$
E_{r 2}^{\prime}=E_{r 2} e^{i \delta_{b}}
$$

Substituting Eqs. (16) and (18) into (7) and (8), and using $H=\sqrt{\frac{\varepsilon_{0}}{\mu_{0}}} n E$, we obtain

$$
\left(\begin{array}{c}
E_{I} \\
H_{I}
\end{array}\right)=M_{B}\left(\begin{array}{c}
E_{I I} \\
H_{I I}
\end{array}\right)
$$

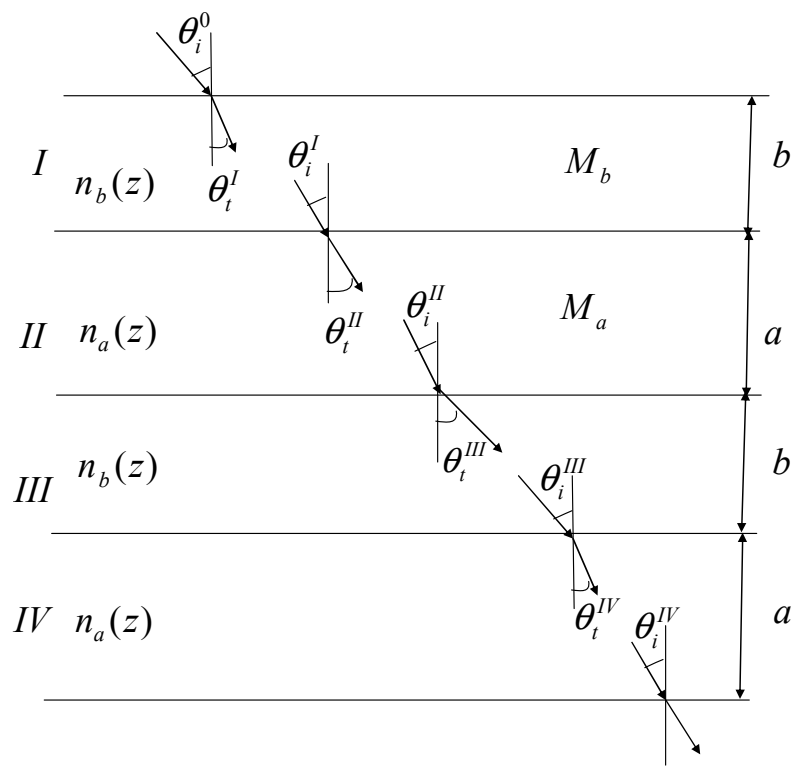

FIG. 4: The two periods transmission figure of light in general function photonic crystals.

where

$M_{B}=\left(\begin{array}{cc}\cos \delta_{b} & -\frac{i \sin \delta_{b}}{\sqrt{\frac{\varepsilon_{0}}{\mu_{0}}} n_{b}(b) \cos \theta_{i}^{I}} \\ -i n_{b}(0) \sqrt{\frac{\varepsilon_{0}}{\mu_{o}}} \cos \theta_{t}^{I} \sin \delta_{b} & \frac{n_{b}(0) \cos \theta_{t}^{I} \cos \delta_{b}}{n_{b}(b) \cos \theta_{i}^{I}}\end{array}\right)$

The Eq. (20) is the transfer matrix $M$ in the medium of FIG. 1 and FIG. 2. By refraction law, we can obtain

$$
\sin \theta_{t}^{I}=\frac{n_{0}}{n(0)} \sin \theta_{i}^{0}, \cos \theta_{t}^{I}=\sqrt{1-\frac{n_{0}^{2}}{n^{2}(0)} \sin ^{2} \theta_{t}^{I}}
$$

where $n_{0}$ is air refractive index, and $n(0)=\left.n(z)\right|_{z=0}$. Using Eqs. (15) and (21), we can calculate $\cos \theta_{i}^{I}$.

4. The structure of one-dimensional general function photonic crystals

In section 3 , we obtain the $M$ matrix of the half period. We know that the conventional photonic crystals is constituted by two different refractive index medium, and the refractive indexes are not continuous on the interface of the two mediums. We could devise the onedimensional general function photonic crystals structure as follows: in the first half period, the refractive index distributing function of medium $B$ is $n_{b}(z)$. and in the second half period, the refractive index distributing function of medium $A$ is $n_{a}(z)$, corresponding thicknesses are $b$ and $a$, respectively. Their refractive indexes satisfy condition $n_{b}(b) \neq n_{a}(0)$, their structure are shown in FIG. 3, and FIG. 4. The Eq. (20) is the half period transfer matrix of medium $B$. Obviously, the half period transfer matrix of medium $\mathrm{A}$ is

$$
M_{A}=\left(\begin{array}{cc}
\cos \delta_{a} & -\frac{i \sin \delta_{a}}{\sqrt{\frac{\varepsilon_{0}}{\mu_{0}}} n_{a}(a) \cos \theta_{i}^{I I}} \\
-i n_{a}(0) \sqrt{\frac{\varepsilon_{0}}{\mu_{o}}} \cos \theta_{t}^{I I} \sin \delta_{a} & \frac{n_{a}(0) \cos \theta_{t}^{I I} \cos \delta_{a}}{n_{a}(a) \cos \theta_{i}^{I I}}
\end{array}\right)
$$


where

$$
\begin{aligned}
& \delta_{a}=\frac{\omega}{c} n_{a}(a)\left[\cos \theta_{i}^{I I} \cdot a\right. \\
& \left.\quad+\sin \theta_{i}^{I I} \int_{0}^{a} \frac{d z}{\sqrt{\frac{n_{a}^{2}(z)}{n_{0}^{2} \sin ^{2} \theta_{i}^{0}}-1}}\right] \\
& \cos \theta_{t}^{I I}=\sqrt{1-\frac{n_{0}^{2}}{n_{a}^{2}(0)} \sin ^{2} \theta_{i}^{0}},
\end{aligned}
$$

and

$$
\begin{gathered}
\sin \theta_{i}^{I I}=\frac{n_{0}}{n_{a}(a)} \sin \theta_{i}^{0}, \\
\cos \theta_{i}^{I I}=\sqrt{1-\frac{n_{0}^{2}}{n_{a}^{2}(a)} \sin ^{2} \theta_{i}^{0}}
\end{gathered}
$$

In one period, the transfer matrix $M$ is

$$
\begin{aligned}
& M=M_{B} \cdot M_{A} \\
& =\left(\begin{array}{cc}
\cos \delta_{b} & \frac{-i \sin \delta_{b}}{\sqrt{\frac{\varepsilon_{0}}{\mu_{0}}} n_{b}(b) \cos \theta_{i}^{I}} \\
-i n_{b}(0) \sqrt{\frac{\varepsilon_{0}}{\mu_{o}}} \cos \theta_{t}^{I} \sin \delta_{b} & \frac{n_{b}(0) \cos \theta_{t}^{I} \cos \delta_{b}}{n_{b}(b) \cos \theta_{i}^{I}}
\end{array}\right) \\
& \left(\begin{array}{cc}
\cos \delta_{a} & \frac{-i \sin \delta_{a}}{\sqrt{\frac{\varepsilon_{0}}{\mu_{0}}} n_{a}(a) \cos \theta_{i}^{I I}} \\
-i n_{a}(0) \sqrt{\frac{\varepsilon_{0}}{\mu_{o}}} \cos \theta_{t}^{I I} \sin \delta_{a} & \frac{n_{a}(0) \cos \theta_{t}^{I I} \cos \delta_{a}}{n_{a}(a) \cos \theta_{i}^{I I}}
\end{array}\right)
\end{aligned}
$$

The form of the GFPCs transfer matrix $M$ is more complex than the conventional PCs. The angle $\theta_{t}^{I}, \theta_{i}^{I}, \theta_{t}^{I I}$ and $\theta_{i}^{I I}$ are shown in Fig. 4. The characteristic equation of GFPCs is

$$
\begin{aligned}
\left(\begin{array}{c}
E_{1} \\
H_{1}
\end{array}\right) & =M_{1} M_{2} \cdots M_{N}\left(\begin{array}{c}
E_{N+1} \\
H_{N+1}
\end{array}\right) \\
& =M_{b} M_{a} M_{b} M_{a} \cdots M_{b} M_{a}\left(\begin{array}{l}
E_{N+1} \\
H_{N+1}
\end{array}\right) \\
& =M\left(\begin{array}{l}
E_{N+1} \\
H_{N+1}
\end{array}\right)=\left(\begin{array}{cc}
A & B \\
C & D
\end{array}\right)\left(\begin{array}{l}
E_{N+1} \\
H_{N+1}
\end{array}\right)(28)
\end{aligned}
$$

Where $N$ is the number of period.

5. The dispersion relation, band gap structure and transmissivity
With the transfer matrix $M$ (Eq. (27)), we can study the dispersion relation and band gap structure of the function photonic crystals.

Since

$$
\left(\begin{array}{c}
E_{N} \\
H_{N}
\end{array}\right)=M\left(\begin{array}{c}
E_{N+1} \\
H_{N+1}
\end{array}\right)=M_{b} M_{a}\left(\begin{array}{c}
E_{N+1} \\
H_{N+1}
\end{array}\right) .
$$

By Bloch law, there is

$$
\left(\begin{array}{c}
E_{N} \\
H_{N}
\end{array}\right)=e^{-i k d}\left(\begin{array}{c}
E_{N+1} \\
H_{N+1}
\end{array}\right)
$$
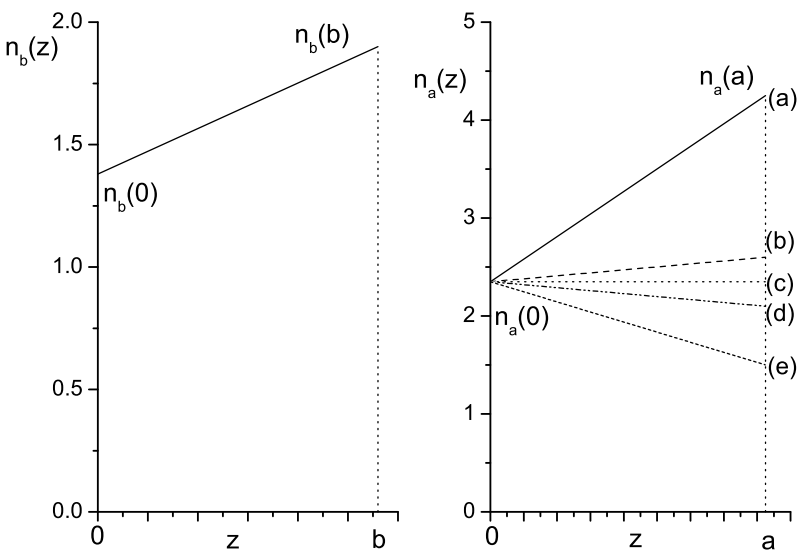

FIG. 5: The line refractive index function in a period. The straight line (a-e) are corresponding to different line functions for medium $A$.

where $d=b+a$. With Eqs. (29) and (30), there is

$$
\left(\begin{array}{c}
E_{N} \\
H_{N}
\end{array}\right)=M_{b} M_{a}\left(\begin{array}{c}
E_{N+1} \\
H_{N+1}
\end{array}\right)=e^{-i k d}\left(\begin{array}{c}
E_{N+1} \\
H_{N+1}
\end{array}\right),
$$

The non-zero solution condition of Eq. (31) is

$$
\operatorname{det}\left(M_{b} M_{a}-e^{-i k d}\right)=0,
$$

i.e.,

$$
\begin{aligned}
& \left(\cos \delta_{b} \cos \delta_{a}-\frac{\eta_{a}}{\eta_{b}} \sin \delta_{b} \sin \delta_{a}-e^{-i k d}\right)\left(\cos \delta_{b} \cos \delta_{a}-\frac{\eta_{b}}{\eta_{a}} \sin \delta_{b} \sin \delta_{a}-e^{-i k d}\right) \\
& +\left(-\frac{i}{\eta_{a}} \cos \delta_{b} \sin \delta_{a}-\frac{i}{\eta_{b}} \sin \delta_{b} \cos \delta_{a}\right)\left(-i \eta_{b} \sin \delta_{b} \cos \delta_{a}-i \eta_{a} \cos \delta_{b} \sin \delta_{a}\right)=0 .
\end{aligned}
$$

Simplifying Eq.(33), we obtain the dispersion relation

$$
\cos k d=\cos \delta_{b} \cos \delta_{a}-\frac{1}{2}\left(\frac{\eta_{b}}{\eta_{a}}+\frac{\eta_{a}}{\eta_{b}}\right) \sin \delta_{b} \sin \delta_{a} .
$$

From Eq. (34), we can study the photonic dispersion re- 


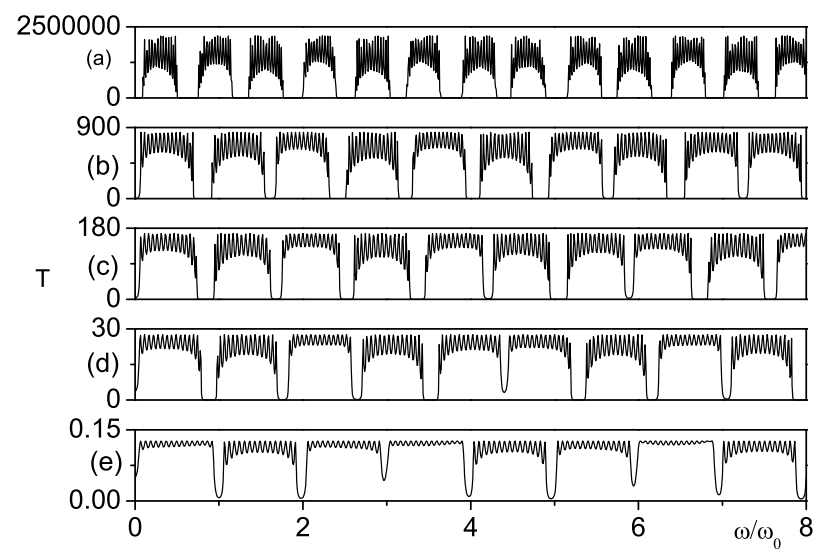

FIG. 6: Comparing the transmissivity of the GFPCs with different line refractive index function: Fig. 6 (a-e) corresponding to Fig. 5 (a-e).

lation and band gap structure, and can obtain the transmission coefficient $t$ from Eq. (28)

$$
t=\frac{E_{t N+1}}{E_{i 1}}=\frac{2 \eta_{0}}{A \eta_{0}+B \eta_{0} \eta_{N+1}+C+D \eta_{N+1}},
$$

and transmissivity $T$ is

$$
T=t \cdot t^{*}
$$

\section{Numerical result}

In this section, we report our numerical results of transmissivity. We consider refractive indexes of the linearity functions in a period, it is

$$
\begin{array}{ll}
n_{b}(z)=n_{b}(0)+\frac{n_{b}(b)-n_{b}(0)}{b} z, & 0 \leq z \leq b, \\
n_{a}(z)=n_{a}(0)+\frac{n_{a}(a)-n_{a}(0)}{a} z, & 0 \leq z \leq a,
\end{array}
$$

Eqs. (37) and (38) are the refractive indexes distribution function of two half period mediums $B$ and $A$, which are shown in FIG. 5. When the endpoint values $n_{b}(0)$, $n_{b}(b), n_{a}(0)$ and $n_{a}(a)$ are all given, the line refractive index functions $n_{b}(z)$ and $n_{a}(z)$ are ascertained.

The main parameters are: the half period thickness $b$ and $a$, the starting point refractive indexes $n_{b}(0)$ and $n_{a}(0)$, and end point refractive indexes $n_{b}(b)$ and $n_{a}(a)$, the optical thickness of the two mediums are equal, i.e., $n_{b}(0) b=n_{a}(0) a$.

In Fig.6, we take $n_{b}(0)=1.38, n_{b}(b)=1.9$, i.e., the refractive index distribution of medium $B$ is confirmable, $n_{a}(0)=2.35$, the incident angle $\theta_{i}^{0}=0$, the center frequency $\omega_{0}=1.215 \times 10^{15} \mathrm{~Hz}, \lambda_{0}=\frac{2 \pi c}{\omega_{0}}$, the thickness $b=280 \mathrm{~nm}$ and $a=165 \mathrm{~nm}$ and the period number
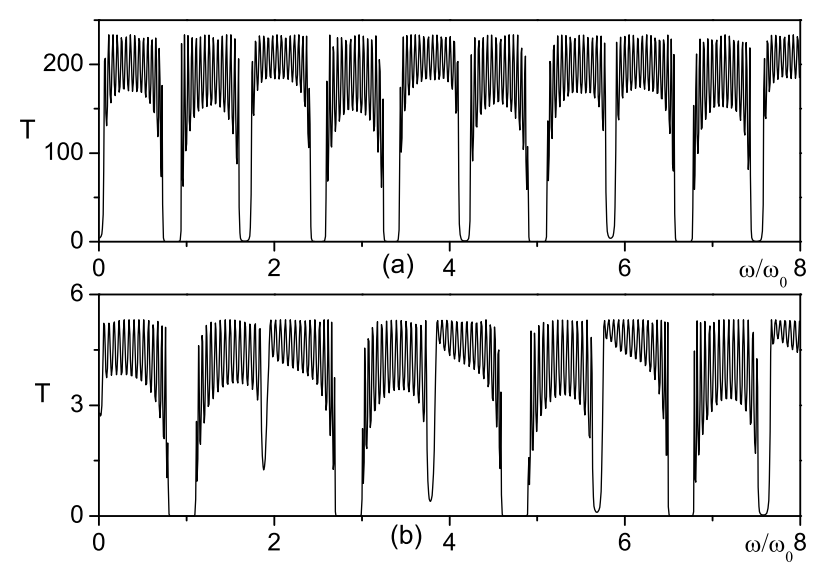

FIG. 7: Comparing the transmissivity of the GFPCs with different line refractive index function for medium $B$.

$N=16$. From Fig. 6(a-e), the end point value of $n_{a}(a)$ are taken as: $4.25,2.6,2.35,2.1$ and 1.5 respectively. And the corresponding refractive indexes distribution are straight lines (a), (b), (c), (d) and (e) in Fig. 5. When the refractive index distribution of medium $B$ maintain unchanging, with the end point value $n_{a}(a)$ of medium $A$ decrease, we can obtain the following results for the onedimensional GFPCs: (1) The maximum of transmissivity decrease. From Fig.6 (a) to (e), their transmissivity maximums are $2500000,900,180,30$ and 0.15 , respectively. (2) The width of band gaps become narrow and the number of band gaps decrease. (3)The transmissivity of one-dimensional GFPCs can be much larger or smaller than 1 for different slope linearity refractive index function, which is different from the transmissivity of conventional $\mathrm{PCs}$ (its transmissivity $T \leq 1$ ). When we take $n_{b} 0=n_{b}(b)$ and $n_{a} 0=n_{a}(a)$, and calculate the transmissivity by the GFPCs equations, i.e., Eqs. (27) and (35), the calculate results of transmissivity is the same as the conventional PCs. So, the conventional PCs is the special case of the GFPCs.

In Fig. 7, we take $n_{a}(0)=2.35, n_{a}(a)=2.4$, i.e., the refractive index distribution of medium $A$ is confirmable, $n_{b}(0)=1.38$, and the end point value $n_{b}(b)$ of medium $B$ are taken as: 1.9 and 1.5. By the two refractive indexes distributions of medium $B$, we obtain the transmissivity are corresponding to Fig. $7(\mathrm{a})$ and (b). It can be found that when the refractive index of medium $A$ keep unchanging, with the end point value $n_{b}(b)$ of medium $B$ decrease, the transmissivity maximum and the number of band gaps decrease, and the width of band gaps increase.

In Fig. 8, Fig. 9 and Fig. 10, the main parameters are: $n_{b}(0)=1.38, n_{b}(b)=1.9, n_{a}(0)=2.35, n_{a}(a)=2.6$. In the inset of Fig. 8, we discuss the influence of period number $N$ on the transmissivity. The results shown in Fig. 8 (a-c) are calculated by the period number $N=12$, 
$N=16$ and $N=20$, respectively. We can see that when the period number of the FGPCs is small, for example, $N=12$ the maximum of transmission intensity achieve 160 [Fig. 8(a)]. When the period number increase up to 20 [Fig. 8(c)], the transmission intensity is nearly 5000, it is much higher than $N=12$, i.e., as the period number increase, the transmission intensity increase.

Figure 9 shows that the effect of different incident angles on transmission intensity. In Fig. 9 (a-d), the incident angles are: $\theta_{i}^{0}=0, \theta_{i}^{0}=\frac{\pi}{6}, \theta_{i}^{0}=\frac{\pi}{4}$ and $\theta_{i}^{0}=\frac{\pi}{3}$, respectively. As we can see in Fig. 9 (a-d), when the incident angle increase, the transmission intensity increase, and the number of band gaps increase.

Figure 10 shows the variation of transmissivity due to the change of the optical thickness. The optical thickness of $A$ and $B$ medium are equal, i.e., $n_{b}(0) b=n_{a}(0) a$. The results shown in Fig. 10 (a-c) are calculated by the optical thickness as: $\frac{\lambda_{0}}{8}, \frac{\lambda_{0}}{4}$ and $\frac{\lambda_{0}}{2}$ respectively. It is found when the optical thickness increase, the number of band gaps increase, while the maximum of transmission intensity keep unchanged.

In the following, we consider two groups line refractive indexes functions in a period, which are all decreasing linearly, they are shown in Fig. 11. The first group main parameters are: $n_{b}(0)=1.9, n_{b}(b)=1.8, n_{a}(0)=2.6$ and $n_{a}(a)=2.5$, it is the solid line (a). The second group main parameters are: $n_{b}(0)=1.5, n_{b}(b)=1.4$, $n_{a}(0)=2.4$ and $n_{a}(a)=2.3$, it is the dash-dot line (b). The optical thickness are same: $n_{b}(0) b=n_{a}(0) a=\frac{\lambda_{0}}{4}$.

Figure 12 is the transmission spectra. The Fig. 12 (a) correspond to the first group line refractive indexes functions, the Fig. 12 (b) correspond to the second group line refractive indexes function. Here, we notice that when the refractive indexes function of the two mediums $A$ and $B$ are all decreasing, the transmission intensity decrease and the width of band gaps become wider.

In Fig. 13-15, the main parameters are: $n_{b}(0)=1.9$, $n_{b}(b)=1.8, n_{a}(0)=2.6$ and $n_{a}(a)=2.5$.

In Fig. 13, we study the effect of period number $N$ on the transmission intensity. The incident angle $\theta_{i}^{0}=0$, the optical thickness $n_{b}(0) b=n_{a}(0) a=\frac{\lambda_{0}}{4}$. They are shown in Fig. 13 (a-c), and the period number are $N=$ $12, N=16$ and $N=20$, respectively. It can be found that as period number $N$ increases from 12 to 20, the transmission intensity decrease.

In addition, we study the transmission intensity with different incident angles as shown in Fig. 14. We take the period number $N=16$, and optical thickness $n_{b}(0) b=n_{a}(0) a=\frac{\lambda_{0}}{4}$. we can find when the incident angle $\theta_{i}^{0}$ increase, the transmission intensity decrease. For example, when the incident angle $\theta_{i}^{0}=0$, the maximum of transmission intensity nearly 0.08 (Fig. 14(a)), and $\theta_{i}^{0}=\frac{\pi}{3}$, the maximum of transmission intensity nearly 0.08 (Fig. 14(d)).

In what follows, we will take into account the transmission at different optical thickness, which is shown in Fig. 15. We also choose $\theta_{i}^{0}=0, N=16$. When the optical thickness are taken $\frac{\lambda_{0}}{8}, \frac{\lambda_{0}}{4}$ and $\frac{\lambda_{0}}{2}$, the transmis-

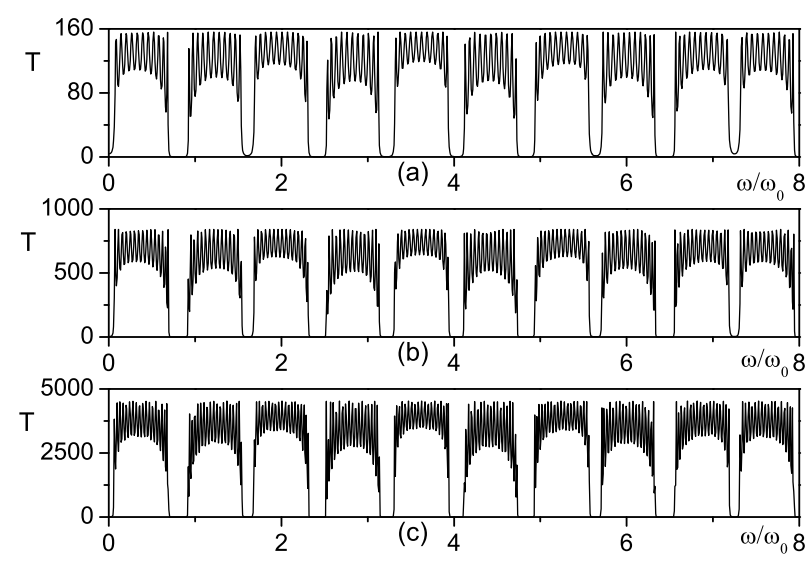

FIG. 8: Comparing the transmissivity of the function PCs with different period number $N$ : (a) $\mathrm{N}=12$, (b) $\mathrm{N}=16$ and (c) $\mathrm{N}=20$.

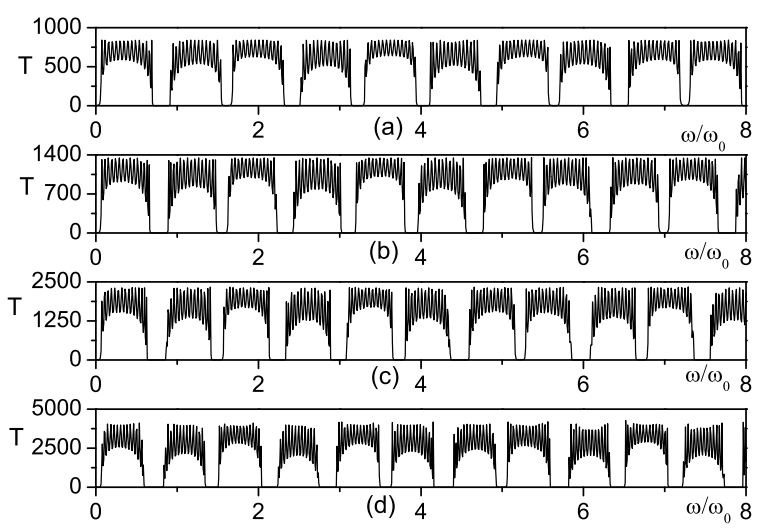

FIG. 9: Comparing the transmissivity of the GFPCs with different incidence angle: (a) $\theta_{i}^{0}=0$, (b) $\theta_{i}^{0}=\frac{\pi}{6}$, (c) $\theta_{i}^{0}=\frac{\pi}{4}$ and (d) $\theta_{i}^{0}=\frac{\pi}{3}$.

sion spectra are corresponding to Fig. 15 (a-c). we can find when the optical thickness increases, the number of band gaps increase, the width of band gaps become narrow, while the maximum of transmission intensity keep unchanged.

\section{Conclusion}

In summary, We have theoretically investigated a new kind of general function photonic crystals (GFPCs), which refractive index is a function of space position. Based on Fermat principle, we achieve the motion equations of light in one-dimensional general function photonic crystals, and calculate its transfer matrix. We choose the linearity refractive index function for two mediums $A$ and $B$, and find the transmissivity of onedimensional GFPCs can be much larger or smaller than 

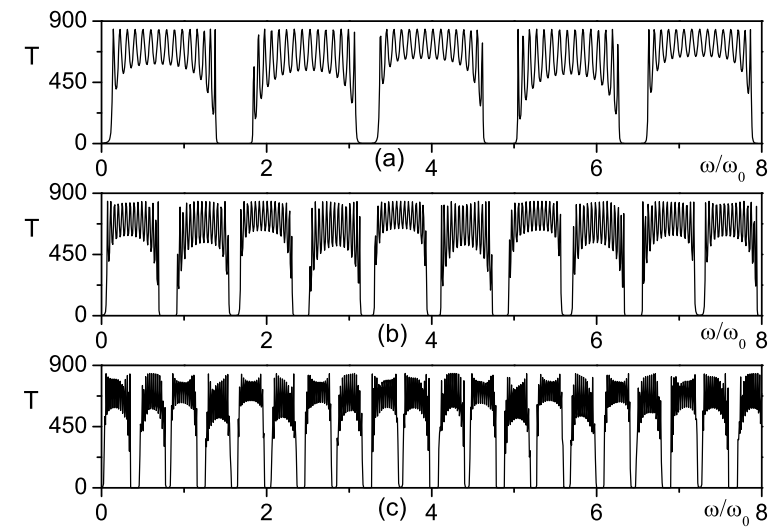

FIG. 10: Comparing the transmissivity of the GFPCs with different optical thickness: (a) $\frac{\lambda_{0}}{8}$, (b) $\frac{\lambda_{0}}{4}$ and (c) $\frac{\lambda_{0}}{2}$.

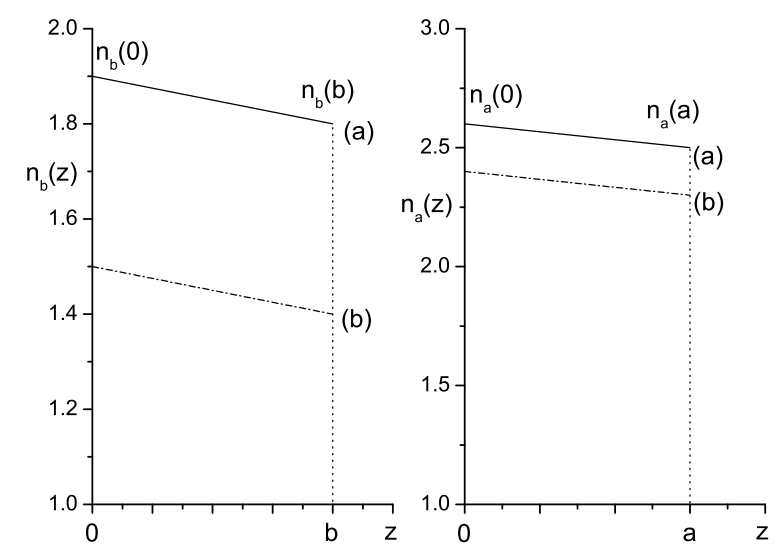

FIG. 11: The two groups line refractive index function in a period: (a) the first group function and (b) the second group function.

1 for different slope linearity refractive index function. Otherwise, we study the effect of different incident angles, the number of periods and optical thickness on the transmissivity, and obtain some new results as follows: (1) when $n_{b}(b)>n_{b}(0)$ and $n_{a}(a)>n_{a}(0)$, the peak value of transmission intensity is much larger than 1 . (2) when $n_{b}(b)<n_{b}(0), n_{a}(a)<n_{a}(0)$, the transmission intensity is much smaller than 1 . With the high and low transmissivity of GFPCs, we can make amplification and decay device of light. (3) For arbitrary medium $A$ or $B$, when its slope of line refractive index function decrease the transmissivity peak value decrease, the number of band gaps decrease and the band gaps become narrow. (4) When the transmissivity $T>1$, as the optical thickness increase, the number of band gaps increase, and its width become narrow, while the peak value of transmissivity is unchanged. (5) When the transmissiv-

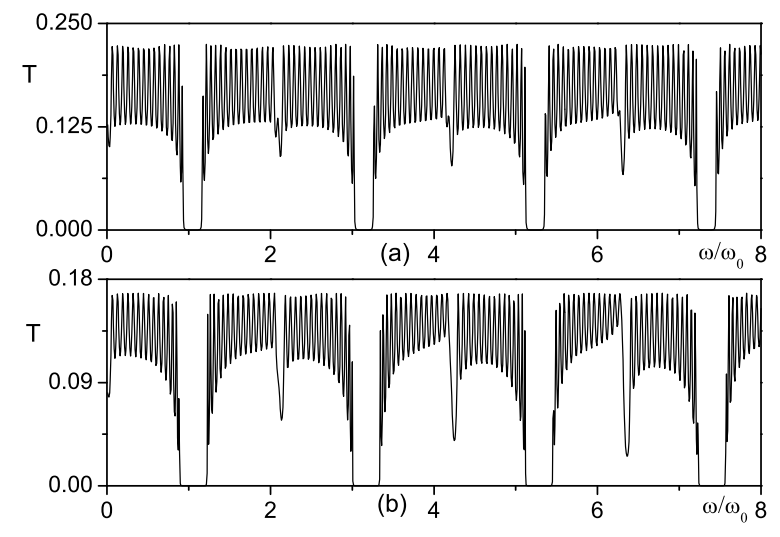

FIG. 12: Comparing the transmissivity of the GFPCs with different line refractive index function: (a) the first group function and (b) the second group function.

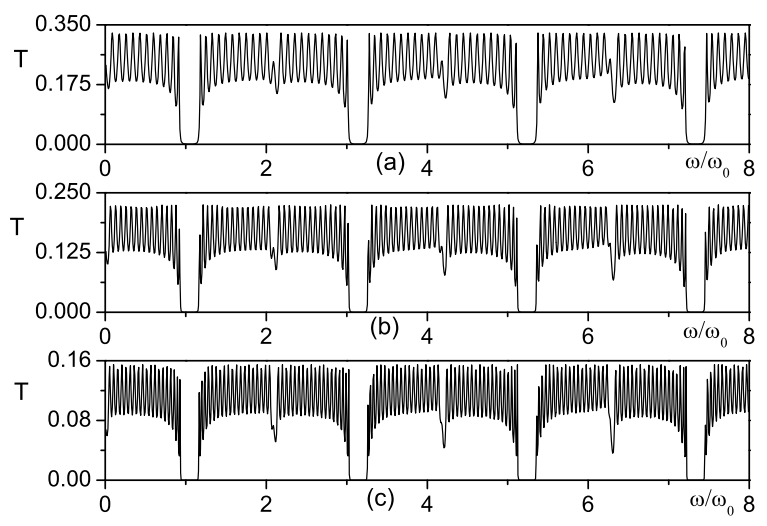

FIG. 13: Comparing the transmissivity of the function PCs with different periodicity (a) $\mathrm{N}=12$ (b) $\mathrm{N}=16$ (c) $\mathrm{N}=20$

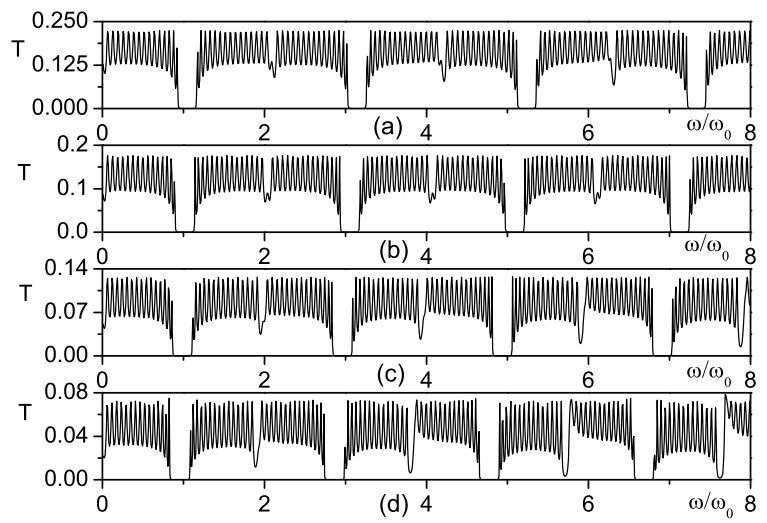

FIG. 14: Comparing the transmissivity of GFPCs with different incidence angles: (a) $\theta_{i}^{0}=0$, (b) $\theta_{i}^{0}=\frac{\pi}{6}$, (c) $\theta_{i}^{0}=\frac{\pi}{4}$ and (d) $\theta_{i}^{0}=\frac{\pi}{3}$. 

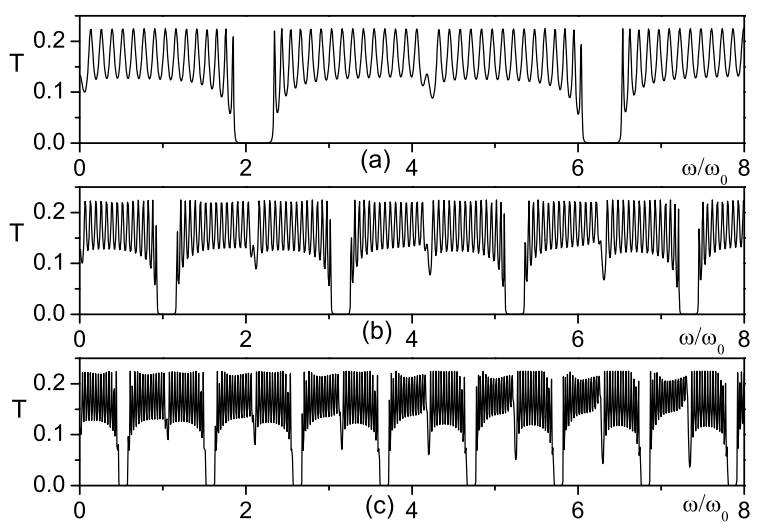

FIG. 15: Comparing the transmissivity of the GFPCs with different optical thickness: (a) $\frac{\lambda_{0}}{8}$, (b) $\frac{\lambda_{0}}{4}$ and (c) $\frac{\lambda_{0}}{2}$. ity $T<1$, as the optical thickness increase, the number of band gaps increase, and its width become narrow, while the peak value of transmissivity is unchanged. (6) When the transmissivity $T>1$, as the incident angles increase, the peak value of transmissivity also increase, and the position of band gaps red shift. (7) When the transmissivity $T<1$, as the incident angles increase, the peak value of transmissivity decrease, and the position of band gaps red shift. (8) When the transmissivity $T>1$, as the number of period increase, the peak value of transmissivity increase, while the position and width of band gaps are unchanged. (9) When the transmissivity $T<1$, as the number of period increase, the peak value of transmissivity decrease, while the position and width of band gaps are unchanged. The new results of GFPCs couldn't be found in conventional PCs, and we think the GFPCs should be widely applied in the future.
[1] E. Yablonovitch, Phys. Rev. Lett. 58, 2059 (1987).

[2] F. Bordas, M. J. Steel, C. Seassal, A. Rahmani., Optics Express. 15, 10890 (2007).

[3] P. Nedel, X. Letartre, C. Seassal, A. Auffves, L. Ferrier, E. Drouard, A. Rahmani, and P. Viktorovitch., Optics Express. 195014 (2011).

[4] C. Zinoni, B. Alloing, L. H. Li, F. Marsili, A. Fiore, L. Lunghi, A. Gerardino, Yu. B. Vakhtomin, K. V. Smirnov, and G. N. Gol'tsman., Appl. Phys. Lett. 91031106 (2007).

[5] S. G. Johnson and J. D. Joannopoulos., Optics Express. 8173 (2001).

[6] S. John, Phys. Rev. Lett. 582486 (1987).

[7] W. C. Stumpf, M. Fujita, M. Yamaguchi, T. Asano and S. Noda., Appl. Phys. Lett. 90231101 (2007).

[8] V. S. C. Manga Rao and S. Hughes., Phys. Rev. Lett. 99 193901 (2007).

[9] G. Lecamp, P. Lalanne, and J. P. Hugonin., Phys. Rev. Lett. 99023902 (2007).

[10] V. S. C. Manga Rao and S. Hughes., Phys. Rev B 75 205437 (2007).

[11] K. Inoue, M. Sasada, J. Kawamata, K. Sakoda, and J. W. Haus, Jpn J. Appl. Phys. Part 238 L157 (1999).

[12] R. D. Meade, A. Devenyi, J. D. Joannopoulos, O. L. Alerhand, D. A. Smith and K. Kash, J. Appl. Phys. 75 4753 (1994).

[13] T. Lund-Hansen, S. Stobbe, B. Julsgaard, H. Thyrrestrup, T. Snner, M. Kamp, A. Forchel, and P. Lodahl., Phys. Rev. Lett. 101113903 (2008).

[14] S. J. Dewhurst, D. Granados, D. J. P. Ellis, A. J. Bennett, R. B. Patel, I. Farrer, D. Anderson, G. A. C. Jones, D. A. Ritchie, and A. J. Shields., Appl. Phys. Lett. 96031109 (2010).

[15] K. Busch and S. John, Phys. Rev. Lett. 83, 967 (1999).

[16] A. Z. Sanchez and P. Halevi, J. Appl. Phys. 94, 797
(2003).

[17] E. Kuramochi, M. Notomi, S. Hughes, A. Shinya, T. Watanabe, and L. Ramunno, Phys. Rev. B72, 161318(R) (2005).

[18] S. Hughes, L. Ramunno, Jeff F. Young, J. E. Sipe., Phys. Rev. Lett. 94033903 (2005).

[19] N. Le Thomas, H. Zhang, J. Jgersk, V. Zabelin, and R. Houdr , I. Sagnes and A. Talneau , Phys. Rev. B80 125332 (2009).

[20] M. Patterson, S. Hughes, S. Combri, N.-V.-Quynh Tran, A. De Rossi, R. Gabet and Y. Jaouen., Phys. Rev. Lett 102253903 (2009).

[21] J-K. Yang, H. Noh, M. J. Rooks, G. S. Solomon, F. Vollmer and H. Cao., Appl. Phys. Lett. 98, 241107 (2011)

[22] A. Figotin, A. Y. Godin and I. Vitebsky, Phys. Rev. B61, 15523 (2000).

[23] R. Martinez-Sala, J. Sancho, J. V. Sanchez, V. Gomez, J. Llinares and F. Meseguer, nature 378, 241 (1995).

[24] J. V. Sanchez-Perez, D. Caballero, R. Martinez-Sala, C. Rubio, J. Sanchez-Dehesa, F. Meseguer, J. Llinares and F. Galvez, Phys. Rev. Lett. 80, 5325 (1998).

[25] J. D. Joannopoulus, S. G. Johnson, J. N. Winn and R. D. Meade, Photonic Crystals. Molding the Flow of Light (Princeton University press, Princeton, 2008).

[26] D. Torrent, A. Hakansson, F. Cervera and J. Sanchez Dehesa, Phys. Rev. Lett. 96, 204302 (2006).

[27] D. Torrent, J. Sanchez-Dehesa, New. Jour. Phys. 9, 323 (2007).

[28] J. O. Vasseur, P. A. Deymier, B. Djafari-Rouhani, Y. Pen - nec and A. C. Hladky-Hennion, Phys. Rev. B 77, 085415 (2008).

[29] Xiang-Yao Wu, Bai-Jun Bai, Jing-Hai Yang, Xiao-Jing Liu, Nuo Ba, Yi-Heng Wu and Qing-Cai Wang, Physica E 43, 1694 (2011). 